\title{
Variação intraspecífica do lenho de Pseudopiptadenia contorta (DC.) G.P. Lewis \& M.P. Lima (Leguminosae - Mimosoideae) de populações ocorrentes em dois remanescentes de Floresta Atlântica
}

\author{
Maria Luiza R. da Costa Ribeiro ${ }^{1}$ e Claudia Franca Barros²,3
}

Recebido em 1/09/2005. Aceito em 5/05/2006

\begin{abstract}
RESUMO - (Variação intraespecífica do lenho de Pseudopiptadenia contorta (DC) G.P. Lewis \& M.P. Lima (Leguminosae - Mimosoidae) ocorrentes em dois remanescentes de Mata Atlântica). O presente trabalho compara populações distintas de Pseudopiptadenia contorta (DC.) G.P. Lewis \& M.P. Lima ocorrentes em dois remanescentes de Floresta Atlântica no Estado do Rio de Janeiro. Foram amostradas árvores de diâmetro semelhante retas e sem defeitos aparentes. Os resultados obtidos comprovam estatisticamente a ocorrência de variação intraspecífica na estrutura anatômica da madeira. Os caracteres qualitativos mantiveram-se constantes, enquanto os quantitativos variaram, sendo os significativos, de acordo com o teste t de Student, a frequiencia, comprimento e diâmetro dos elementos vasos, o comprimento e espessura da parede das fibras, a frequiência e largura dos raios. A análise dos componentes principais, utilizando características anatômicas quantitativas ordenou as duas populações separadamente. O eixo I responde por 33\% da variância total principalmente pela relação positiva do diâmetro do elemento de vaso, enquanto o eixo II responde por $20 \%$ da variância total, principalmente pelo comprimento das fibras.
\end{abstract}

Palavras-chave: Pseudopiptadenia, anatomia da madeira, Leguminosae, variação intraspecífica

\begin{abstract}
Intraspecific variation in wood anatomy of Pseudopiptadenia contorta (DC) G.P. Lewis \& M.P. Lima (Leguminosae Mimosoidae) in two Atlantic rain forest remnants). This study compares distinct populations of Pseudopiptadenia contorta (DC) G.P. Lewis \& M.P. Lima occurring in two remnants of Atlantic rain forest in Rio de Janeiro state. Trees with similar diameters and with no apparent defects were selected. The results confirm intraspecific variation in wood anatomy. Qualitative features do not change, while according to the Student $t$ test quantitative features showed significant differences in vessel-element frequency, width, and length, fiber length and wall thickness, and ray frequency and width. Principal component analysis showed two separate populations. Factor 1 explains $33 \%$ of the total variance, mainly due to the positive relationship of vessel-element tangential diameter; factor 2 explains $20 \%$ of the total variance, mainly due to fiber length.
\end{abstract}

Key words: Pseudopiptadenia, wood anatomy; Leguminosae, intraspecific variation

\section{Introdução}

A família Leguminosae possui 727 gêneros e cerca de 19.325 espécies dos quais cerca de 192 gêneros e 3.270 táxons específicos pertencem à subfamília Mimosoideae (Lewis et al. 2005).

O gênero Pseudopiptadenia Rauschert conta com oito espécies e é exclusivo da faixa neotropical, estendendo-se desde o Norte da América do Sul até o Sul do Brasil. A Floresta Atlântica é o hábitat predominante e, às vezes, exclusivo das espécies do gênero. Pseudopiptadenia contorta (DC.) G.P. Lewis
\& M.P. Lima é freqüente na Floresta Ombrófila Densa tanto nas fisionomias submontanas como montana (Velozo 1992), restingas e matas de cipó, nos Estados da Paraíba, Bahia, Minas Gerais, Rio de Janeiro e São Paulo (Lewis \& Lima 1990). É a espécie de mais ampla distribuição do gênero, bastante característica das matas de encosta e de restinga do estado do Rio de Janeiro (Lewis \& Lima 1990). No Parque Nacional do Itatiaia (PARNA Itatiaia) a espécie ocorre em altitudes de 700 até $900 \mathrm{~m}$, em geral em encostas e no interior da mata (Lewis \& Lima 1990). Em Poço das Antas, a espécie ocorre em Floresta Ombrófila Densa

\footnotetext{
1 Universidade Federal do Rio de Janeiro, Museu Nacional. Programa de Pós-Graduação em Botânica. Trabalho desenvolvido durante a vigência da bolsa PIBIC/CNPq, Programa Mata Atlântica, Instituto de Pesquisas do Jardim Botânico do Rio de Janeiro

2 Instituto de Pesquisas Jardim Botânico do Rio de Janeiro, Programa Mata Atlântica, Rua Pacheco Leão, 915, 22460-030 Rio de Janeiro, RJ, Brasil

3 Autor para correspondência: cbarros@jbrj.gov.br
} 
Submontana (Velozo 1992) onde a topografia é predominantemente de planície, com morros e morrotes de perfis arredondados, cuja altura varia de 30 a $200 \mathrm{~m}$, separados por várzeas alagadiças de fundo achatado (Rôças et al. 2001).

A diversidade estrutural encontrada no xilema secundário das plantas lenhosas tem uma explicação adaptativa e funcional e pode estar diretamente associada ao hábito da planta assim como a variações das condições atmosféricas e à disponibilidade hídrica. A anatomia ecológica da madeira refere-se ao estudo da correlação entre as preferências florísticas e ecológicas do táxon e aos muitos caracteres relativos à função (Dickison 2000). Trabalhos de variação intraespecífica têm demonstrado que as variações ambientais refletem-se em variações estruturais no lenho, principalmente quanto às dimensões dos elementos celulares, tais como: frequiência, diâmetro e comprimento dos elementos de vaso, espessura da parede e comprimento das fibras, altura e largura dos raios (Carlquist \& Hoekman 1985; Barajas-Morales 1985; Wilkins \& Papassotiriou 1989; Arnold \& Mauseth 1999; Noshiro \& Baas 2000). Mesmo considerando que há um número relativamente grande de espécies cujo lenho foi estudado com enfoque ecológico, ainda assim pode-se dizer que este número é pequeno quando se considera a riqueza de espécies da flora brasileira (Marcati et al. 2001).

O presente trabalho tem como objetivo comparar anatomia da madeira de indivíduos de duas populações de Pseudopiptadenia contorta (DC.) G.P. Lewis \& M.P. Lima oriundas de dois remanescentes de Floresta Atlântica do Estado do Rio de Janeiro, verificando e testando estatisticamente a variação intraespecífica.

\section{Material e métodos}

O material botânico estudado é proveniente de dois remanescentes de Floresta Atlântica localizados em duas Unidades de Conservação (Tab. 1) no Estado do Rio de Janeiro: Reserva Biológica de Poço das Antas (22 $30^{\prime}$ e $22^{\circ} 33^{\prime} \mathrm{S}$; $42^{\circ} 15^{\prime}$ e $42^{\circ} 19^{\prime} \mathrm{W}$ ) e Parque Nacional do Itatiaia $\left(22^{\circ} 19^{\prime}\right.$ e $22^{\circ} 45^{\prime} \mathrm{S}$; $44^{\circ} 45^{\prime}$ e $44^{\circ} 50^{\prime} \mathrm{W}$ ).

Todas as amostras coletadas e suas respectivas lâminas encontram-se registradas na Xiloteca do Instituto de Pesquisas Jardim Botânico do Rio de Janeiro (Tab. 2).

Foram selecionadas árvores de tronco cilíndrico, reto e sem bifurcação ou defeito aparente, com diâmetro à altura do peito (DAP) entre $20-30 \mathrm{~cm}$,
Tabela 1. Informações climáticas e geográficas relacionadas aos locais de coleta (Rôças et al. 2001; Guedes-Bruni, comunicação pessoal).

\begin{tabular}{lcc}
\hline & $\begin{array}{c}\text { Reserva Biológica } \\
\text { de Poço das Almas }\end{array}$ & $\begin{array}{c}\text { Parque Nacional } \\
\text { do Itatiaia }\end{array}$ \\
\hline $\begin{array}{l}\text { Localização } \\
\text { Topografia }\end{array}$ & $\begin{array}{c}\text { Silva Jardim } \\
\text { planície } \\
\text { Altitude }\end{array}$ & $\begin{array}{c}\text { Itatiaia } \\
\text { montanhosa }\end{array}$ \\
$\begin{array}{l}\text { Precipitação média anual mar } \\
\text { Temperatura média anual }\end{array}$ & $2.260 \mathrm{~mm}$ & $1.716 \mathrm{~mm}$ \\
Clima & $33^{\circ} \mathrm{C}$ & $21{ }^{\circ} \mathrm{C}$ \\
& Quente e & $\begin{array}{c}\text { Temperado com } \\
\text { estação seca } \\
\text { úmido }\end{array}$ \\
& & (maio a setembro) \\
\hline
\end{tabular}

evitando-se desta forma indivíduos juvenis e variações estruturais decorrentes da comparação entre amostras provenientes de árvores com diâmetro diferente. As amostras foram coletadas por método não destrutivo, com alburno e cerne, com auxílio de serrote, talhadeira e martelo ou do trado marca Sweden modelo S 5. A padronização detalhada das amostras acarreta em uma diminuição do número amostral, como indicado na Tab. 2, mas por outro lado, minimiza o efeito de outras variáveis, como por exemplo diferenças estruturais decorrentes da juvenilidade das árvores coletadas.

A partir das amostras de madeira, foram confeccionados corpos de prova (Coradin \& Muñiz 1991), posteriormente seccionados em micrótomo de deslizamento, nos planos transversal e longitudinal (radial e tangencial), obtendo-se cortes com espessura média de $12 \mu \mathrm{m}$. Após esses procedimentos o material foi clarificado, desidratado e corado em safranina e azul de Astra (Bukastch 1972) para confecção das lâminas permanentes (Johansen 1940). As medições das fibras e do comprimento dos elementos vasculares foram executadas em material dissociado (Jane 1956; Fedalto 1982) e macerado (Dop \& Gautié 1909). As descrições, mensurações e contagens dos elementos

Tabela 2. Relação dos indivíduos analisados, números de registro na Xiloteca do Instituto de Pesquisas Jardim Botânico do Rio de Janeiro (RBw), número de registro no Laminário e local de coleta.

\begin{tabular}{lll}
\hline RBw & Lâmina & Local de coleta da amostra \\
\hline 7701 & 2073 & Parque Nacional do Itatiaia \\
7826 & 2096 & Parque Nacional do Itatiaia \\
7459 & 1888 & Reserva Biológica de Poço das Antas \\
7463 & 1908 & Reserva Biológica de Poço das Antas \\
7464 & 1874 & Reserva Biológica de Poço das Antas \\
\hline
\end{tabular}


celulares obedeceram às normas do IAWA Committee (1989). Para as medições e contagens foi utilizado o software Image Pro-Plus versão 3.0 para Windows acoplado ao microscópio Olympus BH2.

A análise estatística foi realizada com o Software Statística v. 5.0 for Win 95/NT. O teste de ShapiroWilk $\mathrm{W}$ foi utilizado para testar a normalidade das amostras (Zar 1996). Para avaliar o grau de significância da variação, aplicou-se o teste t de Student. Utilizou-se a análise de componentes principais (ACP) para ordenar as populações, assim como para encontrar os componentes de maior variância.

\section{Resultados e discussão}

Os caracteres qualitativos mostraram-se constantes nas duas populações estudadas e concordam com a literatura para Leguminosae, que é caracterizada por apresentar placas de perfuração simples, pontoações alternas guarnecidas, parênquima paratraqueal em diferentes tipos e raios algumas vezes homocelulares (Baas et al. 2000).

Os indivíduos de ambas as populações apresentaram camadas de crescimento distintas, demarcadas pelo achatamento radial e maior espessamento das fibras no lenho tardio e menos freqüentemente pela ocorrência de elementos de vaso de menor diâmetro e dilatação dos raios, características mais evidentes nos indivíduos de Itatiaia (Fig. 1-2). A porosidade é difusa com elementos de vaso solitários, múltiplos de 2-7 elementos e mais raramente em cachos de até 7 elementos, a maioria com apêndice em ambas as extremidades; seção circular a oval. As placas de perfuração são simples. As pontoações intervasculares são guarnecidas, pequenas, alternas, formato poligonal, abertura elíptica e inclusa; pontoações raio-vasculares e parênquimo-vasculares semelhantes às intervasculares. Parênquima axial paratraqueal vasicêntrico, aliforme (mais comum) e confluente; seriado com 1-4 células; presença de séries cristalíferas de até 17 cristais por série (Fig. 6). Os raios são unisseriados, bisseriados (Fig. 3-4) e mais raramente, nos indivíduos de Itatiaia, trisseriados; homogêneos, integrados por células procumbentes (Fig. 5). Não foram encontrados trabalhos sobre a anatomia da madeira de espécies do gênero Pseudopiptadenia. Os resultados observados estão em concordância com Metcalfe \& Chalk (1957), que reporta para a subfamília vasos solitários, em múltiplos ou cachos irregulares, pontoações intervasculares alternas e pequenas, parênquima paratraqueal abundante, ocasionalmente confluente, séries cristalíferas de 11 ou mais cristais e raios homogêneos de 1-9 células de largura.

A análise comparativa entre as populações estudadas evidenciou que os caracteres anatômicos qualitativos mantiveram-se constantes enquanto os quantitativos variaram sob as diferentes condições ambientais dos remanescentes de Floresta Atlântica sendo os significativos, de acordo com o teste $t$ de Student, a frequiência e diâmetro dos elementos de vaso, comprimento e espessura da parede das fibras, a frequiência e altura dos raios (Tab. 3).

A análise dos componentes principais, utilizando características anatômicas quantitativas, ordenou as duas populações separadamente (Fig. 7). O eixo I responde por $33 \%$ da variância total principalmente pela relação positiva do diâmetro dos elementos de vaso, enquanto o eixo II responde por $20 \%$ da variância total, principalmente pelo comprimento das fibras.

Ao serem analisados dados de diferentes altitudes é preciso atentar para o fato de que nem sempre as médias irão crescer ou decrescer constantemente juntamente com a altitude. Para a maior parte dos caracteres como: comprimento das fibras, comprimento dos elementos de vaso e altura dos raios, as médias obedecerão a curvas inconstantes e dependendo da zona altitudinal analisada os valores poderão ser maiores ou menores, como observado por Van der Graaff \& Baas (1974). De acordo com Wheeler \& Baas (1991) com o aumento da altitude (até cerca de $2.000 \mathrm{~m}$ ) os elementos axiais, principalmente elementos de vaso e fibras tenderiam a ficar mais longos e estreitos, tendência observada nas populações estudadas. De acordo com Dickison (2000) e Carlquist (2001) nas dicotiledôneas, caracteres relacionados aos elementos de vaso, como frequiência, comprimento e diâmetro tangencial, são fortemente relacionados às condições ambientais, proporcionando ao mesmo tempo segurança e eficiência no transporte de água e solutos.

Liu \& Noshiro (2003) destacam a ocorrência de variação intraspecífica na anatomia da madeira em espécies de distribuição restrita (confinadas ao hemisfério Norte ou Sul), enquanto as de ampla distribuição (que ocorrem em todos os continentes, em diferentes condições ambientais) não apresentam. Estes resultados foram observados em Acacia melanoxylon R. Br. (Wilkins \& Papassotiriou 1989), Rhododendron anthopogon D. Don, R. lepidotum Wall. ex G. Don, R. campanulatum D. Don e $R$. arboreum Sm. (Noshiro \& Suzuki 1995), Alnus nepalensis D. Don (Noshiro et al. 1994), que apresentaram tendências altitudinais e latitudinais 
842 Ribeiro \& Barros: Variação intraespecífica do lenho de Pseudopiptadenia contorta (DC) G.P. Lewis \& M.P. Lima...
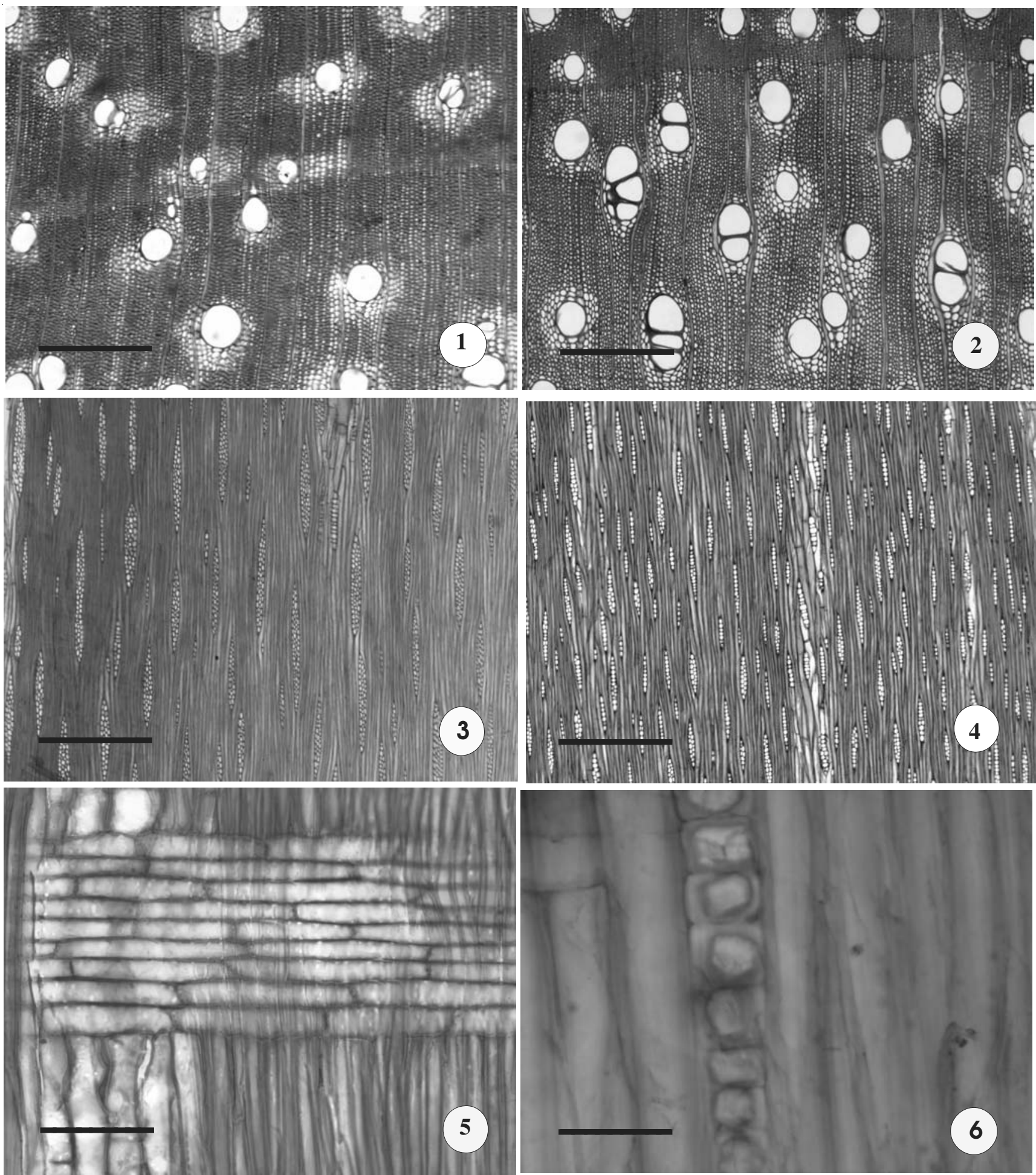

Figura 1-6. 1. Secção transversal de Pseudopiptadenia contorta (DC.) G.P. Lewis \& M.P. Lima ocorrente no Parque Nacional do Itatiaia $($ Barra $=250 \mu \mathrm{m})$. 2. Secção transversal de Pseudopiptadenia contorta (DC.) G.P. Lewis \& M.P. Lima ocorrente na Reserva Biológica de Poço das Antas $($ Barra $=250 \mu \mathrm{m})$. 3. Secção longitudinal tangencial de Pseudopiptadenia contorta (DC.) G.P. Lewis \& M.P. Lima ocorrente no Parque Nacional do Itatiaia $($ Barra $=100 \mu \mathrm{m})$. 4. Secção tangencial longitudinal de Pseudopiptadenia contorta (DC.) G.P. Lewis \& M.P. Lima ocorrente na Reserva Biológica de Poço das Antas (Barra $=100 \mu \mathrm{m})$. 5. Secção radial de Pseudopiptadenia contorta (DC.) G.P. Lewis \& M.P. Lima (Barra $=50 \mu \mathrm{m})$. 6. Secção longitudinal radial de Pseudopiptadenia contorta (DC.) G.P. Lewis \& M.P. Lima evidenciando os cristais (Barra $=50 \mu \mathrm{m})$. 
Tabela 3. Sumário das medições realizadas. Todos os valores foram expressos em $\mu \mathrm{m}$, exceto vasos $/ \mathrm{mm}^{2}$ e raios $/ \mathrm{mm}^{2}$. Valores de $\mathrm{F}$ (significância) assinalados com asterisco apresentaram $\mathrm{P}<0,05$.

\begin{tabular}{|c|c|c|c|c|c|c|c|c|c|}
\hline & \multicolumn{4}{|c|}{$\begin{array}{c}\text { Pseudopiptadenia contorta } \\
\text { (DC.) G.P. Lewis \& M.P. Lima } \\
\text { Reserva Biológica de Poço das Antas }\end{array}$} & \multicolumn{4}{|c|}{$\begin{array}{l}\text { Pseudopiptadenia contorta } \\
\text { (DC.) G.P. Lewis \& M.P. Lima } \\
\text { Parque Nacional do Itatiaia }\end{array}$} & \multirow[t]{2}{*}{$\mathrm{F}$} \\
\hline & Máxima & Média & Mínima & $\begin{array}{l}\text { Desvio } \\
\text { padrão }\end{array}$ & Máxima & Média & Mínima & $\begin{array}{l}\text { Desvio } \\
\text { padrão }\end{array}$ & \\
\hline \multicolumn{10}{|l|}{ Elementos de vaso } \\
\hline Vasos $/ \mathrm{mm}^{2}$ & 24 & 17 & 10 & $\pm 3,96$ & 14,00 & 7,75 & 4,00 & $\pm 2,20$ & $3,25^{*}$ \\
\hline Comprimento & 579,47 & 336,34 & 149,01 & $\pm 103,26$ & 535,01 & 369,09 & 92,81 & $\pm 94,75$ & 1,19 \\
\hline Diâmetro & 170,321 & 108,56 & 70,64 & $\pm 23,00$ & 206,43 & 71,36 & 19,98 & $\pm 38,75$ & $2,84 *$ \\
\hline Espessura da parede & 6,66 & 3,65 & 2,06 & $\pm 0,82$ & 5,99 & 3,55 & 1,86 & $\pm 0,84$ & 1,06 \\
\hline \multicolumn{10}{|l|}{ Pontoações } \\
\hline Intervasculares & 6,40 & 3,88 & 2,56 & $\pm 0,79$ & 5,90 & 4,01 & 2,92 & $\pm 0,75$ & 1,12 \\
\hline Raio-vasculares & 5,94 & 4,11 & 2,61 & $\pm 0,79$ & 5,38 & 4,25 & 2,81 & $\pm 0,63$ & 1,57 \\
\hline Parênquimo-vasculares & 5,73 & 4,04 & 2,42 & $\pm 0,97$ & 5,89 & 4,14 & 3,07 & $\pm 0,66$ & 2,22 \\
\hline \multicolumn{10}{|l|}{ Fibras } \\
\hline Comprimento & $1.333,71$ & 838,26 & 591,00 & $\pm 162,16$ & $1.511,34$ & $1.006,51$ & 615,56 & $\pm 254,35$ & $2,46^{*}$ \\
\hline Parede & 6,25 & 3,84 & 1,88 & $\pm 0,94$ & 10,31 & 5,30 & 2,36 & $\pm 1,80$ & $3,67 *$ \\
\hline \multicolumn{10}{|l|}{ Parênquima radial } \\
\hline Raios $/ \mathrm{mm}^{2}$ & 10,00 & 4,88 & 1,00 & $\pm 1,32$ & 7,00 & 3,78 & 1,00 & $\pm 1,26$ & $1,10^{*}$ \\
\hline Altura & 232,23 & 150,00 & 4 & $\pm 28,20$ & 418,71 & 237,80 & 113,62 & $\pm 70,72$ & $6,29 *$ \\
\hline Largura & 68,15 & 21,41 & 2,57 & $\pm 18,05$ & 50,44 & 22,53 & 7,06 & $\pm 10,74$ & 2,83 \\
\hline
\end{tabular}

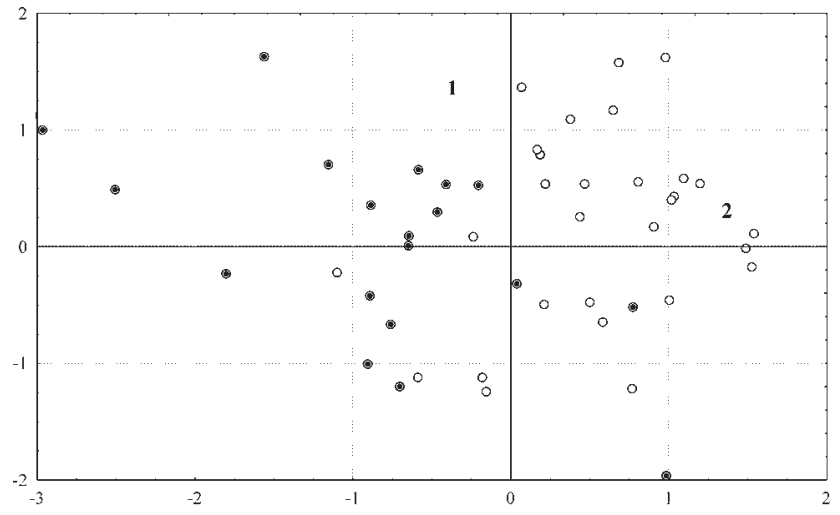

Figura 7. Análise dos componentes principais. 1. diâmetro dos elementos de vaso e 2 . comprimento das fibras. $(\bullet=$ população do Parque Nacional do Itatiaia; 0 = população dåReserva Biológica de Poço das Antas).

significativas e em Dodonaea viscosa Jacq. (Liu \& Noshiro 2003), que não apresentou qualquer correlação entre as variações na anatomia do lenho e nas condições ambientais. Liu \& Noshiro (2003) especulam que provavelmente algumas espécies são geneticamente variáveis enquanto outras possuem um plano mais rígido na estrutura da madeira.

\section{Agradecimentos}

Os autores agradecem ao pesquisador Sebastião José da Silva Neto, pelo auxílio na coleta dos indivíduos; aos pesquisadores Cecília Gonçalves Costa, Micheline Marcon-Ferreira, Carlos Wagner de Oliveira e Natalia Dias de Souza, pela valiosa colaboração no manuscrito; à técnica de laboratório Inês Cosme Neves Grillo, pela confecção das lâminas; ao CNPq, pela concessão de auxílio e bolsa PIBIC; à Petrobrás, pelo patrocínio.

\section{Referências bibliográficas}

Arnold, D.H. \& Mauseth, J.D. 1999. Effects of environmental factors on development of wood. American Journal of Botany 86(3): 367-371.

Barajas-Morales, J. 1985. Wood structural differences between trees of two tropical forests in Mexico. IAWA Bulletin 6(4): 355-364

Baas, P.; Wheller, E. \& Chase, M. 2000. Dycotyledonous wood anatomy and the APG system of angiosperm classification. Botanical Journal of the Linnean Society 131: $3-17$

Bukastch, F. 1972. Bemerkungen zur Doppelfärbung Astrablau-Safranin. Mikrokosmos 61: 1-255. 
Carlquist, S. \& Hoeckman, D.A. 1985. Ecological wood anatomy of the woody southern californian flora. IAWA Bulletin 6(4): 319-347.

Carlquist, S. 2001. Comparative wood anatomy. Systematic, ecological and evolutionary aspects of dycotyledon woods. New York, Springer-Verlag, Berlin, Heidelberg.

Coradin, V.T.R. \& Muniz, G.I.B. 1991. Normas e procedimentos em Estudos de Anatomia de Madeira: I. Angiospermae. II. Gimnospermae. Laboratório de Podutos Florestais - Série Técnica 15: 1-19

Dickison, W.C. 2000. Integrative Plant Anatomy. London, Academic Press.

Dop, D. \& Gautie, A. 1909. Manual de Technique Botanique. Paris, F.R. de Sudeval.

Fedalto, L.C. 1982. Estudo anatomico do lenho de Bixa arborea Huber. Acta Amazonica 12(2): 389-399.

Jane, F.W. 1956. The structure of wood. London, Adam \& Charles Black.

Johansen, D.A. 1940. Plant microtechnique. New York, McGraw Hill Book.

IAWA Commitee. 1989. List of microscopic feature of hardwood identification. IAWA Bulletin 10(3): 219-332.

Lewis, G.P. \& Lima, M.P.M. 1990. Pseudopiptadenia Rauschert no Brasil (Leguminosae-Mimosoidae). Arquivos do Jardim Botânico do Rio de Janeiro 30: 43-68.

Lewis, G.; Schrire, B.; Mackinder, B. \& Lock, M. 2005. Legumes of the World. Kew, Richmond, Royal Botanic Gardens.

Liu, J. \& Noshiro, S. 2003. Lack of latitudinal trends in wood anatomy of Dodonaea viscosa (Sapindaceae), a species with a worldwide distribution. American Journal of Botany 90(4): 532-539.
Marcati, C.R.; Angyalossy-Alfonso, V. \& Benetati, L. 2001. Anatomia comparada do lenho de Copaifera langsdorffii Desf. (Leguminosae-Caesalpinoidae) de floresta e cerradão. Revista Brasileira de Botânica 24(3): 311-320.

Metcalfe, C.R. 1957. Anatomy of the Dicotyledons. $2^{\text {nd }}$ ed. Oxford, Claredon Press.

Noshiro, S.; Joshi, L. \& Suzuki, M. 1994. Ecological wood anatomy of Alnus nepalensis (Betulaceae) in East Nepal. Journal of Plant Research 107: 399-408.

Noshiro, S. \& Suzuki, M. 1995. Ecological wood anatomy of Nepalese Rhododendron (Ericaceae). 2. Intraespecific variation. Journal of Plant Research 108: 217-233.

Noshiro, S. \& Baas, P. 2000. Latitudinal trends in wood anatomy within species and genera: case study in Cornus S.L. (Cornaceae). American Journal of Botany 87(10): 1495-1506.

Rôças, G.; Scarano, F.R. \& Barros, C.F. 2001. Leaf anatomical variation in Alchornea triplinervia (Spreng) Müll. Arg. (Euphorbiaceae) under distinct light and soil water regimes. Botanical Journal of the Linnean Society 136: 231-238.

Van Der Graaff, N.A. \& Baas, P. 1974. Wood anatomical variation in relation to latitude and altitude. Blumea 22: 101-121.

Veloso, H.P. 1992. Manual Técnico da Vegetação Brasileira. Rio de Janeiro, IBGE.

Wheeler, E.A. \& Baas, P. 1991. A survey of the fossil record for dicotyledonous wood and its significance for evolutionary and ecological wood anatomy. IAWA Bulletin 12: 275-332

Wilkins, A.P. \& Papassotiriou, S. 1989. Wood anatomical variation of Acacia melanoxylon in relation to latitude. IAWA Bulletin 10(2): 201-207.

Zar, J.H. 1996. Bioestatistical Analysis. $3^{\text {rd }}$ ed. Prentice Hall. New Jersey. 